\title{
Comparación de la respuesta física, en términos absolutos y relativos a la competición, de diferentes demarcaciones en tareas jugadas de fútbol
}

\section{Comparison of the physical response, in absolute terms and relative to the competition, of different game formats in football taking into account the demarcation of players}

\section{Comparação da resposta física, em termos absolutos e relativos à competição, de diferentes demarcações em tarefas de futebol}

\author{
Zurtuuza, U.; Castellano, J.
}

Facultad de Educación y Deporte, Universidad del País Vasco UPV/EHU, Vitoria-Gasteiz, España

\begin{abstract}
RESUMEN
El objetivo fue estudiar la respuesta física respecto al rendimiento condicional en la competición de diferentes formatos de juego en el fútbol teniendo en cuenta la demarcación de los jugadores. Se registraron cuatro variables de carga externa en 16 jugadores semiprofesionales en cuatro tipos de tareas jugadas (4vs4 [JRP], 6vs6 [JRM] y 8vs8 [JRL]) y partidos de entrenamiento (10vs10 [PE]) para un total de 467 registros. Las variables físicas analizadas fueron las siguientes: (a) DTmin, distancia total en valores absolutos por minuto de actividad, (b) \%DT, distancia total por minuto en valores relativos al perfil de las demandas en competición, (c) PLmin, player load en valores absolutos por minuto de actividad, y (d) \%PL, player load por minuto en valores relativos al perfil de las demandas en competición. Los resultados fueron que en los formatos JRP y JRM para las variables DTmin y PLmin los valores fueron similares entre demarcaciones, pero no siendo igual para las variables \%DT y \%PL. Por el contrario, las diferencias entre las diferentes posiciones de juego se minimizaron en los formatos grandes (JRL y PE) cuando se analizaron las variables \%DT y \%PL, al contrario que para DTmin y PLmin, donde existieron más diferencias entre demarcaciones. La conclusión fue que dependiendo de considerar las variables física en términos absolutos o relativos a la competición las diferencias entre demarcaciones no son uniformes: los formatos reducidos igualan la respuesta condicional en términos absolutos, mientras que los formatos grandes igualan la respuesta condicional entre jugadores en términos al perfil individual en competición.
\end{abstract}

Palabras clave: deporte colectivo, carga externa, tareas jugadas, posición, entrenamiento.

\section{ABSTRACT}

The objective was to study the physical response to conditional performance in competition of different game formats 


\section{Comparación de la respuesta física en tareas futbolísticas de diferentes demarcaciones}

in football taking into account the demarcation of players. Four external load variables were recorded in 16 semiprofessional players in four types of tasks played (4vs4 [JRP], 6vs6 [JRM] and 8vs8 [JRL]) and training matches (10vs10 [PE]) for a total of 467 records. The physical variables analyzed were the following: (a) DTmin, total distance in absolute values per minute of activity, (b) \%DT, total distance per minute in values relative to the profile of demands in competition, (c) PLmin, player load in absolute values per minute of activity, and (d) \%PL, player load per minute in values relative to the profile of demands in competition. The results were that in the JRP and JRM formats for the variables DTmin and PLmin the values were similar between demarcations, but not being the same for the variables \%DT and \%PL. On the contrary, the differences between the different game positions were minimized in the large formats (JRL and PE) when the \%DT and \%PL variables were analyzed, unlike for DTmin and PLmin, where there were more differences between demarcations. The conclusion was that, depending on whether the physical variables are considered in absolute terms or in relation to the competition, the differences between demarcations are not uniform: small formats equal the conditional response in absolute terms, while large formats equal the conditional response between players in terms of the individual profile in competition.

Keywords: collective sport, external load, tasks played, position, training.

\section{RESUMO}

O objetivo era estudar a resposta física ao desempenho condicional na competição de diferentes formatos de jogo no futebol, levando em conta a demarcação dos jogadores. Quatro variáveis de carga externa foram registradas em 16 jogadores semiprofissionais em quatro tipos de tarefas jogadas (4vs4 [JRP], 6vs6 [JRM] e 8vs8 [JRL]) e partidas de treinamento (10vs10 [PE]) para um total de 467 registros. As variáveis físicas analisadas foram as seguintes: (a) DTmin, distância total em valores absolutos por minuto de atividade, (b) \%DT, distância total por minuto em valores relativos ao perfil de exigências na competição, (c) PLmin, carga de jogadores em valores absolutos por minuto de atividade, e (d) \%PL, carga de jogadores por minuto em valores relativos ao perfil de exigências na competição. Os resultados foram que nos formatos JRP e JRM para as variáveis DTmin e PLmin os valores foram semelhantes entre as demarcações, mas não sendo os mesmos para as variáveis \%DT e \%PL. Pelo contrário, as diferenças entre as diferentes posições de jogo foram minimizadas nos grandes formatos (JRL e PE) quando as variáveis \%DT e \%PL foram analisadas, ao contrário do DTmin e PLmin, onde houve mais diferenças entre as demarcações. A conclusão foi que, dependendo se as variáveis físicas são consideradas em termos absolutos ou em relação à competição, as diferenças entre demarcações não são uniformes: formatos pequenos igualam a resposta condicional em termos absolutos, enquanto formatos grandes igualam a resposta condicional entre jogadores em termos do perfil individual em competição.

Palavras chave: esporte coletivo, carga externa, tarefas jogadas, posição, treinamento.

\section{INTRODUCCIÓN}

Una revisión reciente sobre los efectos de entrenar con juegos reducidos concluye que este tipo de tareas generan adaptaciones agudas y crónicas con las que poder optimizar de manera eficaz el rendimiento condicional de jugadores de fútbol (BujalanceMoreno, Latorre-Román y García-Pinillos, 2019). Además, el empleo habitual de este tipo de tareas redunda en la mejora del juego colectivo que están ligados con los aspectos que inciden en el rendimiento de los equipos (Folgado, Gonçalves y Sampaio, 2017; Sarmento et al., 2018), pudiendo incluso tener efectos positivos en la toma de decisiones fuera del ámbito motriz (Aguilar Sánchez, Hernández Mendo, Martín Martínez, Reigal Garrido, y Chirosa Ríos, 2018).
La literatura parece coincidir en que los formatos reducidos favorecen la estimulación de variables fisiológicas (e.g. frecuencia cardiaca o lactato) y neuromusculares (e.g., aceleraciones y desaceleraciones), provocado probablemente por un aumento en la participación de los jugadores sobre el balón, mientras que los formatos más grandes parecen demandar especialmente la dimensión locomotora (e.g. distancia de carrera) y de manera especial las que tienen que ver con velocidades pico o tiempo acumulado en velocidades altas de desplazamiento (Bujalance-Moreno et al., 2019; Casamichana y Castellano, 2010). 


\section{Zurutuza, Castellano.}

Por otro lado, se sabe que existen diferencias en el rendimiento físico en competición en función de la demarcación que los jugadores ocupan dentro del sistema de juego del equipo (Ade, Fitzpatrick y Bradley, 2016; Schuth, Carr, Barnes, Carling y Bradley, 2016). Por tanto, en la búsqueda de la máxima especifidad aplicada en la optimización de los jugadores en el plano condicional podría ser interesante conocer la respuesta física y los efectos a nivel condicional que las tareas jugadas provocan en función de los roles particulares que los jugadores llevan a cabo en el seno de los equipos (SanchezSanchez et al., 2019). Sin embargo, todavía existe una escasa investigación al respecto, encontrándose unos pocos estudios (Dellal et al., 2012; Martín-García, Castellano, Gómez, Cos y Casamichana, 2019) que han atendido esta preocupación.

Una de las principales conclusiones del estudio de Dellal y colaboradores (2012) es que parece que a los defensas centrales les resultó más exigente las tareas de espacio reducido (mayor desplazamiento e intermitencia, probablemente por el hecho de tener que jugar el balón con grandes limitaciones espaciotemporales alejado a lo que están habituados a realizar en los partidos de competición. Por el contrario, el perfil de actividad demandado por este tipo de tareas jugadas podría aproximarse más a la demarcación de las posiciones más adelantadas del sistema de juego, como los delanteros, donde podrían replicarse con más asiduidad los contextos donde deben intervenir durante la competición. Por otro lado, en el trabajo de Martin-García y colaboradores (2019) encontraron que a medida que los formatos de juego fueron más grandes (e.g., mayor espacio de juego y mayor número de jugadores), las demandas de distancia total recorrida y la recorrida por encima de $25 \mathrm{Km} / \mathrm{h}$ aumentaron. No obstante, se incrementan las demandas respecto a la competición en la variable aceleración (ACC) y desaceleración (DEC) cuando se reducen los formatos de juego (e.g., pocos jugadores en un espacio relativo por jugador menor). Sin embargo, en términos relativos a la competición de cada jugador, los formatos de tareas con espacios reducidos (de 5 o 6 jugadores por equipo) se incrementaron las diferencias entre demarcaciones para las variables de aceleración y distancia total.
De manera novedosa se ha comenzado a describir la respuesta física de los jugadores en las sesiones de entrenamiento considerando el perfil competitivo de cada demarcación(Sanchez-Sanchez et al., 2019; Stevens, de Ruiter, Twisk, Savelsbergh y Beek, 2017; Zurutuza, Castellano, Echeazarra y Casamichana, 2017). Esta perspectiva, permite relativizar la carga soportada por los jugadores en el proceso de entrenamiento. Relativizar los valores permite hacer comparable la respuesta física entre jugadores de diferente perfil condicional y contextualizar, por ejemplo, la carga de entrenamiento semanal al compararse con un valor asignado del $100 \%$ a la demanda de la competición (Stevens et al., 2017). Los investigadores (Martín-García et al., 2019) han utilizado esta nueva forma de valorar la carga aplicado a las tareas jugadas e, incluso, abordándose la descripción de los escenarios de máxima exigencia de la competición con lo que tratar de explicar si los formatos jugados pueden replicarlos (Abbott, Brickley y Smeeton, 2018; Lacome, Simpson, Cholley, Lambert y Buchheit, 2018). Afinar en el diseño de tareas de entrenamiento específicas para demarcación permitiría optimizar el rendimiento físico de los jugadores (Martin-García et al., 2019) y reducir las probabilidades de lesión (Gabbett, 2016).

Por todo lo anterior, el objetivo del estudio fue estudiar la respuesta física en términos absolutos y relativos a la competición de diferentes formatos de juego en fútbol teniendo en cuenta las diferentes demarcaciones de los jugadores. Describir los perfiles de actividad relativos a la competición por demarcación en función del tipo de tarea permitirá identificar el grado en el que las tareas replican las demandas particulares de la competición en cada una de las demarcaciones.

\section{MATERIAL Y MÉTODOS}

\section{Diseño}

El presente estudio se realizó en el periodo de competición (enero-abril) de la temporada 2016-17 durante los microciclos del $28^{\mathrm{a}}$ hasta el $37^{\mathrm{a}}$. Todas las sesiones de entrenamiento y los partidos de competición fueron monitorizadas a través de dispositivos de posicionamiento global (GPS) durante los 10 microciclos que duró el estudio. En total, se obtuvieron un total de 467 registros de tarea (Tabla 1) durante 12 sesiones de entrenamiento $(29,2 \pm 10,7$ por jugador) y 81 registros en los 10 partidos de 


\section{Comparación de la respuesta física en tareas futbolísticas de diferentes demarcaciones}

competición ( $5,1 \pm 3,8$ por jugador) a los que se tuvo acceso. Para calcular a cada jugador el valor medio de la demanda en competición de la DT (distancia total) y PL (player load), los valores de partido fueron normalizados a 90 minutos.

\section{Participantes}

En este estudio participaron un total de 20 jugadores de fútbol masculino semiprofesionales del grupo IV de la tercera división en la Liga Española. Los jugadores completan habitualmente entre tres $(270 \mathrm{~min})$ y cuatro (360 min) sesiones de entrenamiento semanales y juegan un partido oficial (90 min) cada fin de semana. Antes de participar en el estudio, todos los jugadores fueron informados sobre el estudio, de los cuales 16 firmaron el consentimiento para poder utilizar sus datos $($ edad $=25,1 \pm 3,7$ años; altura $=178,3 \pm 5,0 \mathrm{~cm}$; peso $=74,6 \pm 7,9 \mathrm{~kg}$; porcentaje de grasa corporal obtenida con la fórmula de Möhr =10,8 $\pm 2,2 \%$ ). Los jugadores fueron diferenciados por la demarcación que ocupan habitualmente en el equipo (Lacome et al., 2018), defensa central (DC), delantero (DEL), defensa lateral (DL), mediocentro (MC) y medio lateral (ML). Los procedimientos éticos utilizados en este estudio se ajustaron a la Declaración de Helsinki, teniendo la aprobación del Comité de Ética de la Universidad del País Vasco (UPV/EHU) con el código M10/2015/303.

\section{Variables de carga externa}

La carga externa de los jugadores fue estudiada a través de las variables de la dimensión locomotora y mecánica: DT y PL. Estas variables ya han sido utilizadas en trabajos anteriores (Casamichana y Castellano, 2010; Zurutuza, Castellano, Echeazarra, Guridi, y Casamichana, 2019). Ambas variables fueron analizadas de dos maneras. Por un lado, en valores absolutos, si bien, debido a la duración diferente de los formatos jugados (ver Tabla 1), todos los valores absolutos se convirtieron en indicador de intensidad, relativo al promedio del minuto de práctica (DTmin y PLmin), es decir, la distancia recorrida o el player load acumulado en el promedio de un minuto de actividad en la tarea. Por otro lado, estas mismas variables también fueron analizadas en términos relativos al porcentaje respecto al perfil competitivo individual de cada jugador (\%DT y \%PL), representando el $100 \%$ el valor para esa variable en la competición particular de cada jugador. De esta manera todos los formatos de juego estudiados, así como el partido de entrenamiento pudieron ser comparables a partir de estos indicadores de intensidad.

\section{Tareas de entrenamiento}

En este estudio se utilizaron tres formatos de juego y los partidos realizados en los entrenamientos: 4vs4 (JRP), 6vs6 (JRM), 8vs8 (JRL), y 10vs10 (PE), donde el número representa el número de jugadores de campo por equipo, excluido el portero. Todas las tareas tuvieron porterías reglamentarias y un portero por equipo. Las características de cada formato de juego están recogidas en la Tabla 1.

\section{Procedimiento}

Nada más comenzar el estudio, los jugadores realizaron un test de velocidad de 40 metros en el mismo campo de entrenamiento y llevando los dispositivos GPS. Además, en el caso de que se detectasen valores superiores en los picos de velocidad, tanto en entrenamiento como en competición, estos fueron tomados como su velocidad pico o máxima del jugador $\left(\mathrm{V}_{\max }\right)$.

La carga externa fue monitorizada utilizando dispositivos GPS S4 (Minimax v.4.0, Catapult Innovations, Victoria, Australia) que funcionan a una frecuencia de muestreo de $10 \mathrm{~Hz}$ y disponen de un acelerómetro triaxial $100 \mathrm{~Hz}$. El dispositivo fue ajustado a la parte trasera superior de cada jugador utilizando un arnés especial. Los dispositivos GPS se activaron 15 min antes del comienzo de cada sesión de entrenamiento o partido, de acuerdo con las instrucciones del fabricante. Antes del inicio del estudio todos los jugadores estaban familiarizados con el uso de dichos dispositivos.

Los datos registrados por los GPS se descargaron posteriormente a un PC para ser analizados con el software Sprint v5.1.4 (Catapult Innovations, Victoria, Australia, 2010). La fiabilidad y la validez de los dispositivos que se utilizaron en este estudio han sido reportadas en trabajos anteriores (Castellano, Casamichana, Calleja-González, San Román y Ostojic, 2011). La media y desviación estándar ( \pm sd) número de satélites durante la recolección de datos fue $12.5( \pm 0.6)$.

Con respecto a las tareas jugadas estudiadas señalar que todos los jugadores estaban familiarizados a ellas ya que fueron habituales en su proceso de entrenamiento. Los investigadores no propusieron 


\section{Zurutuza, Castellano.}

dichas tareas. Finalmente decir, que únicamente los formatos de tarea que fueron seleccionadas para el estudio se llevaron a cabo en los días centrales de las semanas, es decir, alejados siempre más de 48 horas del partido previo y posterior.

\section{Análisis estadístico}

Primeramente, se llevó a cabo un análisis descriptivo de los juegos reducidos (JRP, JRM y JRL) y partidos entrenamientos (PE) utilizando la media y el error standard (SE). Posteriormente, se utilizaron inferencias basadas en la magnitud para analizar los datos, según las recomendaciones de Batterham y Hopkins (2006). Las diferencias entre en los JR y PE en función de la demarcación se evaluaron mediante las diferencias de medias estandarizadas ( $\mathrm{d}$ de Cohen y los límites de confianza en el 90\%). Los umbrales de interpretación para el tamaño del efecto estandarizado (ES) fueron los siguientes (Batterham y Hopkins, 2006): <0,2 (Trivial), 0,2-0,6 (pequeño), 0,6-1,2 (moderado), 1,2-2,0 (largo) y >2,0 (muy largo). El paquete estadístico SPSS v.24.0 para Windows (SPSS Inc, Chicago, IL) y Microsoft Excel fueron utilizados para llevar a cabo los análisis.

\section{RESULTADOS}

Los valores medios y el error standard de cada una de las variables DT y PL en términos absolutos y relativos a la competición, diferenciado por formato de juego y demarcación se muestran en la Figura 1. Con relación a los formatos de juego, las variables DTmin y \%DT fueron más demandadas en los formatos JRL, especialmente, y PE, mientras que las variables PLmin y \%PL fueron la respuesta condicional más requeridas en los formatos de JRM y JRP. Por otro lado, respecto a la demarcación, fue la ML la demarcación que valores más altos mostró en todos los formatos, tanto para la variable DT como para la variable PL.

En la Tabla 2 se recoge la comparativa (a partir del cálculo del tamaño del efecto) entre demarcaciones en cada uno de los JR (JRP, JRM y JRL) y PE en los que participaron. Como se puede apreciar, las diferencias entre las demarcaciones varían en función de si las variables son consideradas en valores absolutos (metros por minuto para la DT y unidades arbitrarias por minuto para el PL), o relativos (porcentaje de DT y PL respecto a la competición de cada jugador). Cabe destacarse que las diferencias son más notables entre demarcaciones cuando son comparadas las variables en términos absolutos que cuando se realiza de manera relativizada a la competición, si bien estas diferencias son particulares en función del tipo de formato. De manera específica, existió un aumento extremadamente grande ( $d$ de Cohen $>2,0$, indicado con una valoración cualitativa de 5) en el formato PE para PLmin en DL vs ML y MC vs ML, en el formato JRL para PLmin en DC vs DEL, DEL vs ML, DL vs ML y MC vs ML, en el formato JRM para PLmin en DC vs ML, DL vs ML y MC vs ML y, finalmente, en el formato JRP para PLmin en DC vs ML, DEL vs ML, DL vs ML y MC vs ML. Existió una disminución extremadamente grande (valor -5) en el formato JRP para DTmin cuando DC, DEL y DL se compararon con ML. En términos relativos solo los formatos intermedios (JRL y JRM) acapararon estas diferencias para la variable \%DT para DC vs ML y para DEL vs ML en ambos formatos, DL vs ML para el formato JRL y MC vs ML para JRM.

\section{DISCUSIÓN}

El objetivo del presente estudio fue describir la respuesta física de jugadores semi-profesionales en cuatro formatos de juego en términos absolutos y relativos a la competición considerando cinco demarcaciones distintas. La principal aportación del estudio es que la interpretación de la respuesta condicional de las diferentes tareas jugadas fue dependiente de si la valoración de la respuesta física, locomotora (DT) y mecánica (PL), fue realizada en términos absolutos o relativos al perfil de la competición particular a cada jugador. Las conclusiones del estudio fueron que a menor número de jugadores por equipo en las tareas existió una mayor similitud en el rendimiento condicional entre demarcaciones en términos absolutos (DTmin y PLmin), mientras que cuando se relativizaron a las demandas particulares de la competición (\%DT y \%PL) fueron los formatos con más jugadores y disputados en dimensiones más grandes donde se dio una mayor similitud de la respuesta física entre demarcaciones.

En la dimensión mecánica y para el conjunto de las demarcaciones, que fue analizada a partir de la variable PL, se resultados se alinearon con los obtenidos por estudios previos (Casamichana y Castellano, 2010; Castellano y Casamichana, 2013). 


\section{Comparación de la respuesta física en tareas futbolísticas de diferentes demarcaciones}

Tareas realizadas en dimensiones reducidas y con pocos efectivos en cada equipo provocó un aumento de la demanda en esta dimensión, disminuyéndose a medida que el espacio relativo al jugador y el número de jugadores por equipo fueron mayores. Por otro lado, e independientemente a la demarcación, en la dimensión locomotora (analizada mediante la variable DT), se pudo constatar que a medida que el número de jugadores por equipo fue mayor la demanda condicional se vio incrementada. En la línea con lo reportado por la literatura, cuanto mayores fueron las dimensiones del terreno de juego, mayores fueron las demandas de desplazamiento (Casamichana y Castellano, 2010; Casamichana, Castellano, GómezDíaz y Martín-García, 2019).

Los resultados del presente trabajo mostraron que las demandas provocadas por los diferentes formatos de juego difieren según la posición que ocupan los jugadores en competición. Dependiendo del tamaño del formato de juego (JRP, JRM, JRL y PE) las diferencias en la respuesta expresada en valores absolutos (en m por min-1) o relativos a la competición (\%) fueron particulares para cada demarcación, coincidiendo con lo propuesto en trabajos anteriores (Abbott et al., 2018; Lacome et al., 2018). Se destaca especialmente que la interpretación de las demandas físicas puede resultar contradictoria según se considere el análisis en términos absolutos o relativos a la competición particular de cada jugador. Esto se refleja en la totalidad de las comparaciones que se han realizado tomando las perspectivas absoluta y relativa (Tabla 2) donde en muy pocos casos la valoración cualitativa resulto igual. En formatos más pequeños (JRP y JRM), caracterizados por disponer de pocos jugadores en un espacio relativo por jugador reducido, la demanda en términos absolutos fue similar entre las diferentes demarcaciones. Sin embargo, cuando se atiende a estas mismas variables, pero en términos relativos a las demandas particulares de la competición, surgen las diferencias entre posiciones. Por el contrario, las diferencias entre las demarcaciones del equipo se minimizan en los formatos más grandes (JRL y PE) cuando se expresaron en términos relativos a la competición, emergiendo las diferencias cuando las variables fueron consideradas en valores absolutos. Desde este punto de vista, podría ser interesante abordar estudios en torno a la gestión de cargas y su relación con la lesión que han tomado gran interés en la literatura especializada (Griffin et al., 2019) a partir de considerar los valores agudos y crónicos de carga desde un punto de vista relativo a la competición individual.

Son varias las limitaciones del estudio que deben contemplarse si se pretende generalizar los resultados. Por un lado, este estudio fue implementado en un único equipo, particularidades en el perfil condicional de los jugadores que ocuparon las demarcaciones en la muestra empleada podría haber condicionado los resultados obtenidos. Sería de gran interés llevar a cabo este estudio con más jugadores y otros equipos, incluso, con diferentes niveles de competencia (e.g., nivel nacional, autonómico o regional). La segunda de las limitaciones tiene que ver con las variables estudiadas, si bien son dos de las variables más empleadas en la literatura para el análisis de la carga de entrenamiento, disponer de otras como distancias recorridas a alta velocidad, aceleraciones y deceleraciones como carga externa, y de manera complementaria, otras variables de carga interna (frecuencia cardiaca o respuesta a la percepción del esfuerzo) permitiría un abordaje más holístico. Finalmente, cabe mencionar, que las características de los formatos de juego que se emplearon para agruparlos no coincidieron en su duración o espacio relativo por jugador, lo cual podría haber condicionado los resultados obtenidos. A pesar de las limitaciones, los resultados del presente estudio sugieren que los cuatro formatos de tareas estudiadas estimulan de manera dispar a las demarcaciones, pudiéndose ser implementadas en el proceso de intervención para sobre-estimular, replicar o subestimular la respuesta física teniendo como referencia el perfil condicional particular que cada demarcación/jugador tiene en la competición.

\section{CONCLUSIONES}

La conclusión del estudio fue que no todas las tareas estudiadas (JRP, JRM, JRL y PE) demandaron por igual distancia total y carga mecánica, tanto en términos absolutos como relativos a la competición, a todas las demarcaciones (DC, DL, MC, ML y DEL) en el fútbol semi-profesional. Por lo tanto, se debería atender a las particularidades de las demarcaciones cuando se diseñan tareas de entrenamiento para, en función del objetivo, poder replicar, supraestimula o infraestimular las variables condicionales que mejor se ajusten al perfil particular de competición. Las 


\section{Zurutuza, Castellano.}

investigaciones futuras deberían centrarse más en el conocimiento de las demandas de este tipo de tareas considerando las demandas individuales de la competición que cada demarcación tiene en el equipo con la que optimizar el proceso de entrenamiento y en consecuencia su rendimiento condicional.

\section{APLICACIONES PRÁCTICAS}

La principal aplicación de los resultados del estudio es que el uso combinado de los formatos de tareas de juego, desde pequeñas hasta largas, en el proceso de entrenamiento permitiría sobre-estimular o subestimular la respuesta física de los jugadores en cuanto a distancia recorrida y carga mecánica. Por esta razón, parece aconsejable que los entrenadores consideren todo el espectro de tareas (e.g., desde 1vs1 a 10vs10) para atender a las necesidades particulares de cada demarcación y contextualizado al momento del día de la semana y periodo de la temporada con el propósito de optimizar su rendimiento.

\section{REFERENCIAS}

1. Abbott, W., Brickley, G., y Smeeton, N. J. (2018). Positional differences in GPS outputs and perceived exertion during soccer training games and competition. Journal of Strength Conditioning Research, 32(11), 3222-3231. DOI: 10.1519/JSC.0000000000002387.

2. Ade, J., Fitzpatrick, J., y Bradley P. S. (2016). Highintensity efforts in elite soccer matches and associated movement patterns, technical skills and tactical actions. Information for position-specific training drills. Journal of Sports Sciences, 34(24), 2205-2214. DOI:

https://doi.org/10.1080/02640414.2016.1217343.

3. Aguilar Sánchez, J., Hernández Mendo, A., Martín Martínez, I., Reigal Garrido, R. E., y Chirosa Ríos, L. J. (2018). Efectos de un programa de juegos reducidos sobre la toma de decisiones en chicas adolescentes. Cuadernos De Psicología Del Deporte, 18(1), 21-30. Recuperado a partir de https://revistas.um.es/cpd/article/view/295891.

4. Batterham, A. M., y Hopkins, W. G. (2006). Making inferences about magnitudes. International Journal of Sports Physiology and Performance, 1, 50-57.
5. Bujalance-Moreno, P., Latorre-Román, P. Á., y García-Pinillos, F. (2019). A systematic review on small-sided games in football players: Acute and chronic adaptations. Journal of Sports Sciences, 37(8), 921-949.

DOI: https://doi.org/10.1080/02640414.2018.1535821.

6. Casamichana, D., y Castellano, J. (2010). Timemotion, heart rate, perceptual and motor behaviour demands in small-sides soccer games: Effects of pitch size. Journal of Sports Sciences, 28(14), 1615-1623. DOI: $10.1080 / 02640414.2010 .521168$.

7. Casamichana, D., Castellano, J., Gómez-Díaz, A., y Martín-García, A. (2019). Looking for complementary intensity variables in different training games in football. Journal of Strength Conditioning Research, 5. DOI: 10.1519/JSC.0000000000003025.

8. Castellano, J., Casamichana, D., Calleja-González, J., San Román, J., y Ostojic, S. (2011). Reliability and accuracy of $10 \mathrm{~Hz}$ GPS devices for short-distance exercise. Journal of Sports Science and Medicine, 10(1), 233-234.

9. Castellano, J. y Casamichana, D. (2013). Differences in the number of accelerations between small-sided games and friendly matches in soccer. Journal of Sports Science and Medicine, 12(1), 209210.

10. Dellal, A., Owen, A., Wong, D. P., Krustrup, P., van Exsel, M., y Mallo, J. (2012). Technical and physical demands of small vs. large sided games in relation to playing position in elite soccer. Human Movement Science, 31(4), 957-969. DOI: 10.1016/j.humov.2011.08.013. Epub 2012 Feb 17.

11. Lacome, M., Simpson, B. M., Cholley, Y., Lambert, P., y Buchheit, M. (2018). Small-Sided Games in Elite Soccer: Does One Size Fit All? International Journal of Sports Physiology and Performance, 13(5), 568-576. DOI: 10.1123/ijspp.2017-0214.

12. Gabbett, T. J. (2016). The training-injury prevention paradox: should athletes be training smarter and harder? British Journal of Sports Medicine, 50(5), 273-280. doi: 10.1136/bjsports2015-095788. 


\section{Comparación de la respuesta física en tareas futbolísticas de diferentes demarcaciones}

13. Folgado, H., Gonçalves, B., y Sampaio, J. (2017). Positional synchronization affects physical and physiological responses to preseason in professional football (soccer). Research in Sports Medicine, O(0), $1-13$.

14. Griffin, A., Kenny, I. C., Comyns, T. M., Lyons, M., Griffin, A., y Kenny, I. C. (2019). The association between the acute : chronic workload ratio and injury and its application in team sports : a systematic review. Sports Medicine, (0123456789). https://doi.org/10.1007/s40279-019-01218-2

15. Martín-García, A., Castellano, J., Gómez, A., Cos, F., y Casamichana, D. (2019). Positional demands for various-sided games with goalkeepers according to the most demanding passages of match play in football. Biology of Sport, 36(2), 171-180. DOI: 10.5114/biolsport.2019.83507.

16. Sanchez-Sanchez, J., Sanchez, M., Hernández, D., Gonzalo-Skok, O., Casamichana, D., RamirezCampillo, R., y Nakamura, F. Y. (2019). Physical performance during soccer-7 competition and smallsided games in U12 players. Journal of Human Kinetics, 67, 281-290. DOI: 10.2478/hukin-20180082.

17. Sarmento, H., Clemente, F. M., Harper, L. D., Costa, I. T. da, Owen, A., y Figueiredo, A. J. (2018). Small sided games in soccer-a systematic review. International Journal of Performance Analysis in Sport, 18(5), 693-749. https://doi.org/10.1080/24748668.2018.1517288

18. Schuth, G., Carr, C., Barnes, C., Carling, C., y Bradley P. S. (2016). Positional interchanges influence the physical and technical match performance variables of elite soccer players. Journal of Sports Sciences, 34(6), 501-508. DOI: https://doi.org/10.1080/02640414.2015.1127402.

19. Stevens, T. G. A., de Ruiter, C. J., Twisk, J. W. R., Savelsbergh, G. J. P., y Beek, P. J. (2017). Quantification of in-season training load relative to match load in professional Dutch Eredivisie football players. Science and Medicine in Football, 1, 117125.

DOI:

https://doi.org/10.1080/24733938.2017.1282163.
20. Zurutuza, U., Castellano, J., Echeazarra, I., y Casamichana, D. (2017). Absolute and relative training load and its relation to fatigue in football. Frontiers in Psychology, 8, 878. DOI: 10.3389/fpsyg.2017.00878.

21. Zurutuza, U., Castellano, J., Echeazarra, I., Guridi, I., y Casamichana, D. (2019). Selecting training-load measures to explain variability in football training games. Frontiers in Psychology. https://doi.org/10.3389/fpsyg.2017.00878. 


\section{Zurutuza, Castellano.}

Tabla 1. Descripción de las características de las cuatro tareas de juego.

\begin{tabular}{rccccc}
\hline Tarea & $\begin{array}{c}\text { Número de jugadores } \\
\text { por equipo }\end{array}$ & $\begin{array}{c}\text { Espacio relativo } \\
\text { por jugador }\left(\mathrm{m}_{2}\right)\end{array}$ & Repeticiones & $\begin{array}{c}\text { Duración } \\
(\mathrm{min}: \mathrm{sec})\end{array}$ & $\begin{array}{c}\text { Número de } \\
\text { registros }\end{array}$ \\
\hline PE & 10 & 300,0 & 2 & $18: 42$ & 88 \\
JRL & 8 & 268,8 & 2 & $12: 48$ & 130 \\
JRM & 6 & 129,8 & 3 & $8: 36$ & 28 \\
JRP & 4 & 132,7 & 4 & $2: 48$ & 221 \\
\hline
\end{tabular}

Nota: juego reducido pequeño (JRP), juego reducido mediano (JRM), juego reducido largo (JRL) y partido de entrenamiento (PE).

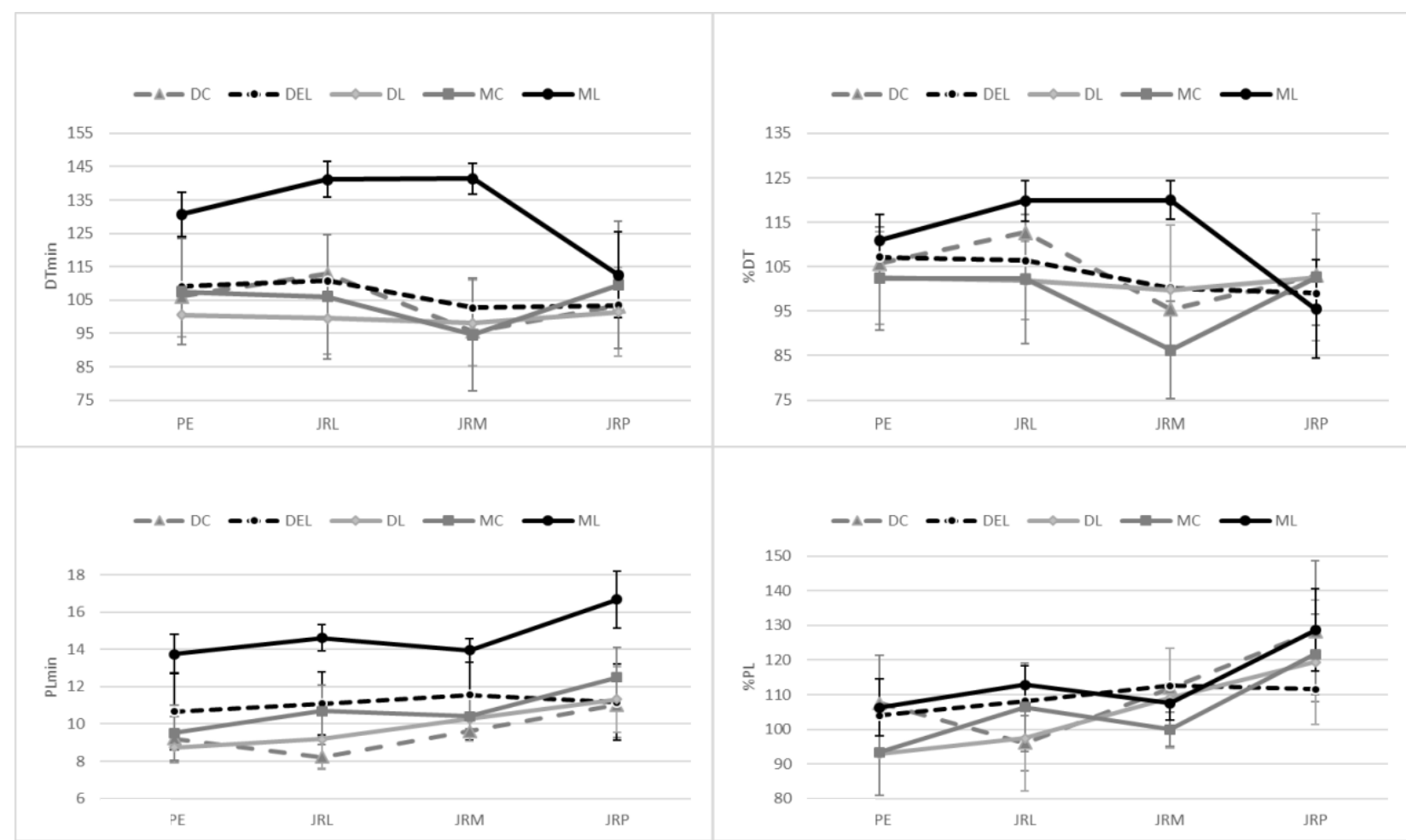

Figura 1. La media y el error estándar de cada una de las variables en términos absolutos, DTmin y PLmin, y relativos a la competición, \%DT y \%PL, diferenciado por formato de juego y demarcación.

Nota: JRP es 4vs4 (cuatro jugadores por equipo), JRM es 6vs6, JRL es 8vs8, and PE es 10vs10. DTmin es distancia total recorrida en valores absolutos, \%DT es distancia total recorrida en valores relativos, PLmin es player load analizado en valores absolutos y \%PL es player load analizado en valores relativos. DC es defensa central, DEL es delantero, DL es defensa lateral, MC es medio centro, ML es medio lateral. 


\section{Comparación de la respuesta física en tareas futbolísticas de diferentes demarcaciones}

Tabla 2. Tamaños del efecto ( $d$ de Cohen), error standard (SE) y límite inferior (LL) y superior (UL) en la comparativa entre demarcaciones diferenciado por tareas.

\begin{tabular}{|c|c|c|c|c|c|c|}
\hline Tarea & Demarcación & Variable & $\begin{array}{c}\text { Absoluto (por min) } \\
{[\text { d de Cohen + SE (LL/UL) }]}\end{array}$ & VC & $\begin{array}{c}\text { Relativo }(\%) \\
{[d \text { de } \text { Cohen }+ \text { SE }(\mathrm{LL} / \mathrm{UL})]}\end{array}$ & VC \\
\hline \multirow{20}{*}{$\mathrm{PE}$} & DCvsDEL & DT & $-0,20 \pm 0,55(-1,10 / 0,71)$ & 0 & $0,11 \pm 0,55(-0,79 / 1,02)$ & 1 \\
\hline & DCvsDEL & PL & $0,88 \pm 0,57(-0,04 / 1,81)$ & 3 & $-0,26 \pm 0,55(-1,16 / 0,65)$ & -1 \\
\hline & DCvsDL & DT & $0,51 \pm 0,62(-0,51 / 1,53)$ & 2 & $-0,31 \pm 0,62(-1,32 / 0,70)$ & -1 \\
\hline & DCvsDL & PL & $-0,44 \pm 0,62(-1,46 / 0,57)$ & -1 & $-1,13 \pm 0,65(-2,20 /-0,06)$ & -2 \\
\hline & DCvsMC & DT & $-0,69 \pm 0,59(-1,66 / 0,28)$ & -2 & $-0,30 \pm 0,58(-1,25 / 0,65)$ & -1 \\
\hline & DCvsMC & PL & $0,24 \pm 0,58(-0,71 / 1,19)$ & 2 & $-1,06 \pm 0,61(-2,06 /-0,07)$ & -2 \\
\hline & DCvsML & DT & $-2,46 \pm 0,94(-4,00 /-0,93)$ & -4 & $0,61 \pm 0,72(-0,57 / 1,80)$ & 3 \\
\hline & DCvsML & PL & $4,07 \pm 1,24(2,04 / 6,10)$ & 4 & $-0,11 \pm 0,71(-1,27 / 1,05)$ & 0 \\
\hline & DELvsDL & DT & $0,84 \pm 0,44(0,12 / 1,56)$ & 3 & $-0,37 \pm 0,42(-1,07 / 0,33)$ & -1 \\
\hline & DELvsDL & PL & $-1,24 \pm 0,45(-1,98 /-0,49)$ & -3 & $-0,86 \pm 0,44(-1,58 /-0,15)$ & -2 \\
\hline & DELvsMC & DT & $-0,71 \pm 0,38(-1,33 /-0,09)$ & -2 & $-0,36 \pm 0,37(-0,97 / 0,25)$ & -1 \\
\hline & DELvsMC & PL & $-0,64 \pm 0,38(-1,26 /-0,03)$ & -2 & $-0,80 \pm 0,38(-1,43 /-0,18)$ & -2 \\
\hline & DELvsML & DT & $-2,60 \pm 0,67(-3,70 /-1,50)$ & -4 & $0,34 \pm 0,55(-0,57 / 1,25)$ & 2 \\
\hline & DELvsML & PL & $1,89 \pm 0,62(0,88 / 2,90)$ & 4 & $0,20 \pm 0,55(-0,70 / 1,11)$ & 2 \\
\hline & DLvsMC & DT & $-1,26 \pm 0,50(-2,08 /-0,44)$ & -3 & $-0,01 \pm 0,46(-0,76 / 0,74)$ & 0 \\
\hline & DLvsMC & PL & $0,64 \pm 0,47(-0,12 / 1,41)$ & 3 & $0,04 \pm 0,46(-0,70 / 0,79)$ & 1 \\
\hline & DLvsML & DT & $-3,91 \pm 1,01(-5,56 /-2,26)$ & -4 & $1,01 \pm 0,65(-0,05 / 2,07)$ & 3 \\
\hline & DLvsML & PL & $5,32 \pm 1,25(3,28 / 7,36)$ & 5 & $1,30 \pm 0,67(0,21 / 2,40)$ & 4 \\
\hline & MCvsML & DT & $-1,53 \pm 0,64(-2,58 /-0,48)$ & -3 & $0,94 \pm 0,60(-0,05 / 1,92)$ & 3 \\
\hline & MCvsML & PL & $3,28 \pm 0,82(1,94 / 4,62)$ & 5 & $1,21 \pm 0,62(0,20 / 2,22)$ & 4 \\
\hline \multirow{20}{*}{ JRL } & DCvsDEL & DT & $-0,48 \pm 0,63(-1,50 / 0,55)$ & -1 & $-0,44 \pm 0,62(-1,46 / 0,59)$ & -1 \\
\hline & DCvsDEL & PL & $2,22 \pm 0,71(1,06 / 3,38)$ & 5 & $1,29 \pm 0,65(0,22 / 2,35)$ & 4 \\
\hline & DCvsDL & DT & $0,20 \pm 0,69(-0,93 / 1,34)$ & 2 & $-0,74 \pm 0,71(-1,90 / 0,43)$ & -2 \\
\hline & DCvsDL & PL & $0,80 \pm 0,71(-0,37 / 1,97)$ & 3 & $0,12 \pm 0,69(-1,01 / 1,25)$ & 1 \\
\hline & DCvsMC & DT & $-0,36 \pm 0,68(-1,48 / 0,76)$ & -1 & $-0,62 \pm 0,69(-1,75 / 0,51)$ & -2 \\
\hline & DCvsMC & PL & $2,25 \pm 0,83(0,89 / 3,62)$ & 5 & $0,97 \pm 0,71(-0,19 / 2,13)$ & 3 \\
\hline & DCvsML & DT & $-1,90 \pm 0,87(-3,32 /-0,47)$ & -3 & $0,52 \pm 0,74(-0,69 / 1,74)$ & 2 \\
\hline & DCvsML & PL & $9,46 \pm 2,48(5,40 / 13,52)$ & 5 & $2,45 \pm 0,95(0,89 / 4,01)$ & 5 \\
\hline & DELvsDL & DT & $1,12 \pm 0,47(0,35 / 1,89)$ & 3 & $-0,53 \pm 0,45(-1,27 / 0,20)$ & -1 \\
\hline & DELvsDL & PL & $-1,17 \pm 0,47(-1,94 /-0,40)$ & -2 & $-0,82 \pm 0,46(-1,57 /-0,07)$ & -2 \\
\hline & DELvsMC & DT & $-0,08 \pm 0,42(-0,77 / 0,61)$ & 0 & $-0,35 \pm 0,42(-1,05 / 0,35)$ & -1 \\
\hline & DELvsMC & PL & $-0,25 \pm 0,42(-0,94 / 0,45)$ & -1 & $-0,15 \pm 0,42(-0,85 / 0,54)$ & 0 \\
\hline & DELvsML & DT & $-2,32 \pm 0,60(-3,31 /-1,33)$ & -4 & $2,03 \pm 0,58(1,08 / 2,99)$ & 5 \\
\hline & DELvsML & PL & $2,73 \pm 0,64(1,68 / 3,77)$ & 5 & $0,54 \pm 0,51(-0,30 / 1,37)$ & 2 \\
\hline & DLvsMC & DT & $-0,76 \pm 0,54(-1,64 / 0,12)$ & -2 & $0,03 \pm 0,52(-0,82 / 0,88)$ & 1 \\
\hline & DLvsMC & PL & $1,02 \pm 0,55(0,12 / 1,93)$ & 3 & $0,64 \pm 0,53(-0,23 / 1,51)$ & 3 \\
\hline & DLvsML & DT & $-2,36 \pm 0,76(-3,61 /-1,12)$ & -4 & $2,56 \pm 0,79(1,28 / 3,85)$ & 5 \\
\hline & DLvsML & PL & $4,51 \pm 1,09(2,72 / 6,30)$ & 5 & $1,35 \pm 0,65(0,29 / 2,41)$ & 4 \\
\hline & MCvsML & DT & $-1,34 \pm 0,63(-2,37 /-0,31)$ & -3 & $1,63 \pm 0,65(0,56 / 2,70)$ & 4 \\
\hline & MCvsML & PL & $3,54 \pm 0,90(2,06 / 5,01)$ & 5 & $0,65 \pm 0,58(-0,31 / 1,61)$ & 3 \\
\hline \multirow{11}{*}{ JRM } & DCvsDEL & DT & $-0,25 \pm 0,78(-1,52 / 1,03)$ & -1 & $0,49 \pm 0,78(-0,79 / 1,77)$ & 2 \\
\hline & DCvsDEL & PL & $1,15 \pm 0,81(-0,18 / 2,47)$ & 3 & $0,06 \pm 0,77(-1,21 / 1,33)$ & 1 \\
\hline & DCvsDL & DT & $0,16 \pm 0,87(-1,27 / 1,58)$ & 1 & $0,36 \pm 0,87(-1,07 / 1,79)$ & 2 \\
\hline & DCvsDL & PL & $0,79 \pm 0,90(-0,67 / 2,26)$ & 3 & $-0,29 \pm 0,87(-1,72 / 1,13)$ & -1 \\
\hline & DCvsMC & DT & $-1,00 \pm 0,91(-2,50 / 0,49)$ & -2 & $-0,97 \pm 0,91(-2,47 / 0,52)$ & -2 \\
\hline & DCvsMC & PL & $1,10 \pm 0,92(-0,42 / 2,61)$ & 3 & $-3,29 \pm 1,28(-5,39 /-1,18)$ & -4 \\
\hline & DCvsML & DT & $-1,42 \pm 1,12(-3,25 / 0,42)$ & -3 & $3,91 \pm 1,71(1,11 / 6,71)$ & 5 \\
\hline & DCvsML & PL & $9,44 \pm 3,49(3,73 / 15,16)$ & 5 & $-1,24 \pm 1,09(-3,03 / 0,55)$ & -3 \\
\hline & DELvsDL & DT & $0,55 \pm 0,60(-0,44 / 1,53)$ & 2 & $-0,03 \pm 0,59(-1,00 / 0,94)$ & 0 \\
\hline & DELvsDL & PL & $-0,67 \pm 0,61(-1,67 / 0,32)$ & -2 & $-0,25 \pm 0,59(-1,22 / 0,73)$ & -1 \\
\hline & DELvsMC & DT & $-1,16 \pm 0,63(-2,19 /-0,13)$ & -2 & $-1,26 \pm 0,64(-2,31 /-0,22)$ & -3 \\
\hline
\end{tabular}




\section{Zurutuza, Castellano.}

\begin{tabular}{|c|c|c|c|c|c|c|}
\hline & DELvsMC & PL & $-0,62 \pm 0,60(-1,61 / 0,37)$ & -2 & $-1,14 \pm 0,63(-2,17 /-0,11)$ & -2 \\
\hline & DELvsML & DT & $-1,80 \pm 0,86(-3,21 /-0,40)$ & -3 & $2,35 \pm 0,91(0,86 / 3,85)$ & 5 \\
\hline & DELvsML & PL & $1,37 \pm 0,82(0,02 / 2,72)$ & 4 & $-0,46 \pm 0,78(-1,74 / 0,82)$ & -1 \\
\hline & DLvsMC & DT & $-1,11 \pm 0,76(-2,36 / 0,13)$ & -2 & $-1,05 \pm 0,75(-2,28 / 0,19)$ & -2 \\
\hline & DLvsMC & PL & $0,11 \pm 0,71(-1,05 / 1,27)$ & 1 & $-0,84 \pm 0,74(-2,04 / 0,37)$ & -2 \\
\hline & DLvsML & DT & $-1,96 \pm 1,03(-3,65 /-0,26)$ & -3 & $1,88 \pm 1,02(0,21 / 3,56)$ & 4 \\
\hline & DLvsML & PL & $3,84 \pm 1,41(1,54 / 6,15)$ & 5 & $-0,14 \pm 0,87(-1,56 / 1,28)$ & 0 \\
\hline & MCvsML & DT & $0,20 \pm 0,87(-1,23 / 1,62)$ & 1 & $4,06 \pm 1,46(1,67 / 6,45)$ & 5 \\
\hline & MCvsML & $\mathrm{PL}$ & $4,17 \pm 1,48(1,74 / 6,60)$ & 5 & $1,51 \pm 0,97(-0,08 / 3,10)$ & 4 \\
\hline \multirow{20}{*}{ JRP } & DCvsDEL & DT & $-0,78 \pm 0,33(-1,33 /-0,24)$ & -2 & $-0,24 \pm 0,33(-0,78 / 0,29)$ & -1 \\
\hline & DCvsDEL & PL & $0,08 \pm 0,32(-0,45 / 0,61)$ & 1 & $-0,78 \pm 0,33(-1,33 /-0,24)$ & -2 \\
\hline & DCvsDL & DT & $0,34 \pm 0,33(-0,21 / 0,88)$ & 2 & $-0,03 \pm 0,33(-0,57 / 0,52)$ & 0 \\
\hline & DCvsDL & PL & $0,17 \pm 0,33(-0,37 / 0,72)$ & 1 & $-0,46 \pm 0,34(-1,01 / 0,09)$ & -1 \\
\hline & DCvsMC & DT & $-1,15 \pm 0,38(-1,77 /-0,53)$ & -2 & $-0,03 \pm 0,35(-0,61 / 0,55)$ & 0 \\
\hline & DCvsMC & PL & $0,89 \pm 0,37(0,29 / 1,50)$ & 3 & $-0,40 \pm 0,36(-0,98 / 0,19)$ & -1 \\
\hline & DCvsML & DT & $-3,37 \pm 0,58(-4,32 /-2,42)$ & -5 & $-0,61 \pm 0,39(-1,24 / 0,02)$ & -2 \\
\hline & DCvsML & PL & $3,46 \pm 0,59(2,49 / 4,43)$ & 5 & $0,03 \pm 0,38(-0,59 / 0,65)$ & 1 \\
\hline & DELvsDL & DT & $1,61 \pm 0,26(1,19 / 2,03)$ & 4 & $0,21 \pm 0,22(-0,15 / 0,58)$ & 2 \\
\hline & DELvsDL & PL & $0,08 \pm 0,22(-0,29 / 0,44)$ & 1 & $0,39 \pm 0,22(0,02 / 0,75)$ & 2 \\
\hline & DELvsMC & DT & $-0,46 \pm 0,26(-0,88 /-0,04)$ & -1 & $0,23 \pm 0,25(-0,18 / 0,65)$ & 2 \\
\hline & DELvsMC & PL & $0,72 \pm 0,26(0,30 / 1,15)$ & 3 & $0,57 \pm 0,26(0,15 / 0,99)$ & 2 \\
\hline & DELvsML & DT & $-3,05 \pm 0,39(-3,70 /-2,40)$ & -5 & $-0,24 \pm 0,29(-0,70 / 0,23)$ & -1 \\
\hline & DELvsML & PL & $3,04 \pm 0,39(2,40 / 3,69)$ & 5 & $0,97 \pm 0,30(0,48 / 1,45)$ & 3 \\
\hline & DLvsMC & DT & $-1,87 \pm 0,31(-2,38 /-1,36)$ & -3 & $0,00 \pm 0,26(-0,43 / 0,43)$ & 0 \\
\hline & DLvsMC & PL & $0,71 \pm 0,27(0,27 / 1,15)$ & 3 & $0,15 \pm 0,26(-0,28 / 0,58)$ & 1 \\
\hline & DLvsML & DT & $-4,68 \pm 0,54(-5,56 /-3,80)$ & -5 & $-0,56 \pm 0,30(-1,05 /-0,07)$ & -1 \\
\hline & DLvsML & PL & $3,25 \pm 0,43(2,55 / 3,96)$ & 5 & $0,62 \pm 0,30(0,13 / 1,11)$ & 3 \\
\hline & MCvsML & DT & $-2,62 \pm 0,43(-3,32 /-1,91)$ & -4 & $-0,65 \pm 0,33(-1,19 /-0,12)$ & -2 \\
\hline & MCvsML & PL & $2,65 \pm 0,43(1,94 / 3,36)$ & 5 & $0,60 \pm 0,32(0,07 / 1,13)$ & 2 \\
\hline
\end{tabular}

Nota: JRP es 4vs4 (cuatro jugadores por equipo), JRM es 6vs6, JRL es 8vs8, and PE es 10vs10. DT es distancia total cubierta y PL es player load. DC es defensa central, DEL es delantero, DL es defensa lateral, MC es medio centro, ML es medio lateral. VC es valoración cualitativa. 0 es trivial, -1 es pequeña disminución, -2 es disminución moderada, -3 es gran disminución, -4 es muy grande disminución, -5 es extremadamente grande disminución, 1 es pequeño aumento, 2 es aumento moderado, 3 es gran aumento, 4 es aumento muy grande y 5 es aumento extremadamente grande. 